\title{
Activation of Nrf2 signaling as a common treatment of neurodegenerative diseases
}
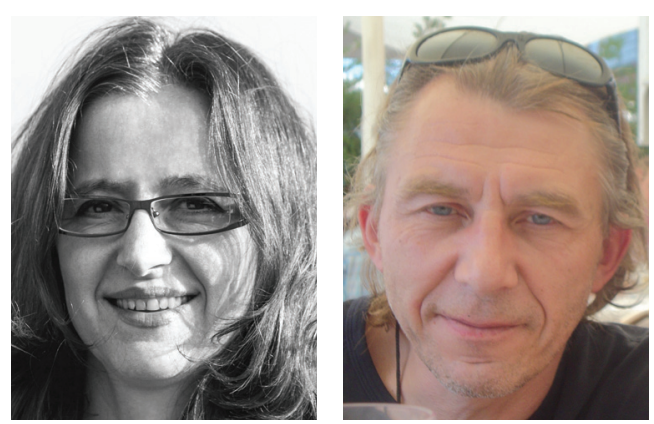

Albena T Dinkova-Kostova ${ }^{1,2}$ \& Aleksey G Kazantsev ${ }^{*, 3}$ “...although the clinical manifestations of neurodegenerative diseases are distinct, these disorders share similar molecular mechanisms of neurodegeneration, which

could be counteracted by common treatment with Nrf2 activators."

First draft submitted: 7 February 2016; Accepted for publication: 7 March 2017; Published online: 23 May 2017

Devastating, age-dependent neurodegenerative disorders, including prevalent disorders such as Alzheimer's disease (AD) and Parkinson's disease (PD), as well as rare disorders such as Huntington's disease (HD) and amyotrophic lateral sclerosis (ALS), affect millions of people worldwide. Their prevalence is projected to increase due to aging of the population, with a corresponding increase in the associated socioeconomic impact. Currently, there is no disease-modifying therapy for any neurodegenerative disease. Symptomatic treatments are available for some conditions such as PD and HD, but the therapeutic benefits are transient and limited. Although the causative factors and clinical manifestations are distinct for each neurodegenerative disease, their molecular pathogeneses share common underlying factors, such as excessive levels of reactive oxygen species (ROS), largely due to mitochondrial impairment, neuroinflammation and disturbances in protein homeostasis (proteostasis). This raises the exciting possibility for developing a universal treatment, targeting these common drivers of neurodegeneration.

The transcription factor, Nrf2, orchestrates a major endogenous defense mechanism against oxidative and xenobiotic stress and inflammation, and plays a role in the maintenance of mitochondrial function and cellular proteostasis, suggesting potential benefits of therapeutic targeting of Nrf2 to counteract neurodegeneration. Under stress condition(s), Nrf2 activates the transcriptional upregulation of a large network of cytoprotective genes, allowing adaptation and survival [1]. The levels (and activity) of Nrf2 are tightly regulated through ubiquitination and proteasomal degradation mediated by several ubiquitin ligase systems, including

\section{KEYWORDS}

neurodegenerative disease

- neuroinflammation •

neuroprotection • Nrf2 signaling

- oxidative stress 


\author{
“The neuroprotective \\ potential of Nrf2 activation \\ has been evaluated in \\ experiments using genetic \\ mouse Huntington's \\ disease models."
}

Keap1-Cul3/Rbx1, $\beta$-TrCP-Cul1 and Hrd1 [2]. Of these, Keap1 is the best understood key negative regulator of $\mathrm{Nrf} 2$.

Keap1 serves as a sensor for electrophiles and oxidants, which chemically modify specific cysteines in Keap1, resulting in conformational changes that protect Nrf2 from Keap1-directed degradation. As a result, Nrf2 accumulates, translocates to the nucleus, binds (as a heterodimer with a small Maf transcription factor) to antioxidant response elements in the promoter of its target genes and activates the expression of a large network of detoxification, antioxidant and anti-inflammatory genes as well as genes involved in clearance of damaged proteins [1]. Of particular interest is upregulation of genes responsible in biosynthesis and regeneration of glutathione (GSH), a major intracellular antioxidant. Nrf2 also suppresses proinflammatory responses, in part through transcriptional repression [3], and is involved in the maintenance of mitochondrial function [4]. Keap1 and p62/SQSTM1 are Nrf2responsive proteins and principal regulators of negative and positive feedback loop mechanisms, respectively. Additionally, p62 targets Keap1 for selective degradation through autophagy [5], thus contributing to sustained Nrf2 activation response.

Aging is associated with increase in ROS and chronic inflammation, suggesting a loss of adaptability and/or impairment of Nrf2 signaling, which are particularly pronounced in age-dependent neurodegenerative diseases [6]. Intriguingly, rare mutations in SQSTM1 cause susceptibility to the human neurodegenerative condition, ALS and frontotemporal lobar degeneration, and are associated with muted Nrf2 activation responses in disease models [7]. Studies suggest a reciprocal relationship and show negative influence of mutant disease-causative proteins on $\mathrm{Nrf2}$ signaling, thereby implicating inhibition of the Nrf2 pathway as a possible mechanism underlying severity and progression of neurodegeneration.

ALS, an adult-onset neurodegenerative disease caused by selective death of motor neurons in the brain and spinal cord, is characterized by progressive muscle weakness and atrophy and is uniformly fatal, usually within 5 years of clinical presentation [8,9]. ALS has a predominant sporadic ALS form with no apparent genetic component, however approximately $5-10 \%$ clinical cases display an autosomal dominant inheritance pattern or familial form of disease, termed as fALS, with known gene causative mutations. The clinical features of sporadic ALS and familial ALS are remarkably similar, suggesting involvement of common pathogenic mechanisms like oxidative stress and neuroinflammation.

Collective work elucidated the oxidative stress and neuroinflammation as the key therapeutic targets of Nrf2 signaling in ALS [10]. Genetic studies in ALS mouse models have shown a significant therapeutic effect of elevated Nrf2 levels in astrocytes, the major GSH suppliers for neighboring neurons. Moreover, Nrf2 signaling is critical for attenuating neuroinflammation in ALS through repression of the deleterious effects of activated microglia on neuronal survival. Consistent with the therapeutic potential of Nrf2 signaling, treatment with small molecule activators, including the extremely potent cyanoenone triterpenoids has shown efficacy in mouse ALS models [11].

The neuroprotective potential of Nrf2 activation has been evaluated in experiments using genetic mouse HD models. HD is an autosomal dominant and highly penetrant neurodegenerative disorder, which results from the pathological expansion of a trinucleotide CAG repeats encoding polyglutamines in HTT protein [12]. Brains from HD patients typically display marked striatal and cortical atrophy at the time of diagnosis. Once motor or other symptoms become apparent, typically during midlife, the affected individuals become increasingly disabled over the course of 15-25 years before succumbing to the effects of severe physical and mental deterioration.

Complex pathogenic mechanisms have been implicated in HD, however excessive oxidative stress has been recognized as an important driver of pathology [13]. The harmful role of oxidative stress has been described in both HD patients and in experimental models, and is potentially due to inherent neuronal sensitivity to an excess of ROS. The levels of several Nrf2-dependent antioxidant proteins including glutathione peroxidases, catalase and superoxide dismutase 1 are increased in human HD brains as compared with non-disease controls, suggesting a partial activation of Nrf2 defense signaling, yet insufficient to block progressive neurodegeneration. Consistent with that notion, pharmacological activation of Nrf2 induces broad antioxidant effects in HD mouse brain and ameliorates the neurological phenotype [10]. Elevated expression of several key inflammatory mediators has been 
observed in blood, striatum, cortex and cerebellum from postmortem patient HD tissues, however neuroinflammation in HD appears to be less pronounced than in ALS or PD.

Similarly, the neurological phenotype of the more prevalent neurodegenerative disease, PD, can be ameliorated by Nrf2 activation [14]. PD is characterized by progressive loss of dopaminergic neurons in the substantia nigra and profound reduction of dopamine in the striatum. Currently available dopaminergic treatments offer partial symptomatic relief and only address the motor manifestations. Multiple genetic and environmental factors have been implicated in PD etiology, however, similar to ALS, the majority of the clinical cases are sporadic. The discovery, that the environmental neurotoxin 1-methyl-4-phenyl-1,2,3,6-tetrahydropyridine (MPTP) causes Parkinsonism in humans, led to the development of the MPTP mouse model of disease, which to date remains a highly utilized animal model of sporadic PD, including evaluation of drug efficacy [15]. Nrf2 activators showed neuroprotective effects in MPTP mice, which are associated with a reduction of oxidative damage and neuroinflammation [15]. The identification of causative mutations in SNCA, the gene encoding $\alpha$-synuclein (aSyn), paved the way for developing genetic mouse PD models, in which daily oral delivery of the Nrf2 activator dimethyl fumarate (DMF) protected nigral dopaminergic neurons against aSyn toxicity [16] .

Although oxidative stress and neuroinflammation constitute pathological hallmarks of AD, a therapeutic role of $\mathrm{Nrf} 2$ signaling has emerged more slowly, perhaps due to the complexity of disease pathogenesis and readouts of efficacy. Nevertheless there is a number of recent publications which demonstrate the efficacy of Nrf2 activators in AD mouse models [17].

DMF, a US FDA-approved drug (Tecfidera, Biogen-Idec) for the treatment of relapsing multiple sclerosis [18], activates Nrf2 through covalent modification of the Keap1 sensor. DMF is of relatively low potency and specificity, which prevents its broad repurposing for neurodegenerative indications. Drug-like molecules with similar mechanism of action or with ability of direct interference with the Keap1/Nrf2 interaction are emerging $[19,20]$. The available data demonstrate the feasibility to develop Nrf2 activators for clinical use.

In summary, although the clinical manifestations of neurodegenerative diseases are distinct, these disorders share similar molecular mechanisms of neurodegeneration, which could be counteracted by common treatment with Nrf2 activators. Moreover, targeting Nrf2 signaling may provide an exciting therapeutic option to ameliorate disease pathology in majority of clinical cases with unknown etiology. Because pharmacological Nrf2 activation targets broad mechanisms of disease, all neurodegenerative conditions would be eligible for therapy. Therefore a major goal is to develop noninvasive oral treatment(s) for outpatient settings under supervision of primary care physicians, which targets both sporadic and familial patients. With simple and inexpensive drug synthesis and manufacturing and a straightforward treatment regimen, the medical cost is expected to be low, with meaningful impact on disease burden and survival. It is also expected that $\mathrm{Nrf} 2$ activating drugs would be self-administered, avoiding additional clinical visits, except those incurred from a longer life expectancy. For clinicians, it would revolutionize the approach to therapy and provide a meaningful treatment option where few have ever existed and entire practices would be reworked to ensure that all patients are offered therapy immediately after diagnosis.

\section{Financial \& competing interests disclosure}

The authors have no relevant affliations or financial involvement with any organization or entity with a financial interest in or financial conflict with the subject matter or materials discussed in the manuscript. This includes employment, consultancies, honoraria, stock ownership or options, expert testimony, grants or patents received or pending, or royalties.

No writing assistance was utilized in the production of this manuscript.

\section{References}

1 Hayes JD, Dinkova-Kostova AT. The Nrf2 regulatory network provides an interface between redox and intermediary metabolism. Trends Biochem. Sci. 39(4), 199-218 (2014).
2 Harder B, Jiang T, Wu T, Tao S et al. Molecular mechanisms of $\mathrm{Nrf} 2$ regulation and how these influence chemical modulation for disease intervention. Biochem. Soc. Trans. 43(4), 680-686 (2015).
3 Kobayashi EH, Suzuki T, Funayama R et al. Nrf2 suppresses macrophage inflammatory response by blocking proinflammatory cytokine transcription. Nat. Commun. 23(7), 11624 (2016). 
4 Holmström KM, Kostov RV, DinkovaKostova AT. The multifaceted role of Nrf2 in mitochondrial function. Curr. Opin. Toxicol. 1, 80-91 (2016).

5 Taguchi K, Fujikawa N, Komatsu M et al. Keap1 degradation by autophagy for the maintenance of redox homeostasis. Proc. Natl Acad. Sci. USA 109(34), 13561-13566 (2012).

6 Corenblum MJ, Ray S, Remley QW et al. Reduced Nrf2 expression mediates the decline in neural stem cell function during a critical middle-age period. Aging Cell 15(4), 725-736 (2016).

7 Goode A, Rea S, Sultana M et al. ALS-FTLD associated mutations of SQSTM1 impact on Keap1-Nrf2 signalling. Mol. Cell. Neurosci. 76, 52-58 (2016).

8 Sreedharan J, Brown RH. Amyotrophic lateral sclerosis: problems and prospects. Ann. Neurol. 74(3), 309-316 (2013).

9 Turner MR, Bowser R, Bruijn L et al. Mechanisms, models and biomarkers in amyotrophic lateral sclerosis. Amyotroph. Lateral Scler. Frontotemporal Degener. 14(Suppl. 1), 19-32 (2013).

10 Johnson DA, Johnson JA. Nrf2 a therapeutic target for the treatment of neurodegenerative diseases. Free Radic. Biol. Med. 88(Pt B), 253-267 (2015).
11 Kanno T, Tanaka K, Yanagisawa Y et al. A novel small molecule, $\mathrm{N}$-(4-(2-pyridyl) (1,3-thiazol-2-yl))-2-(2,4,6trimethylphenoxy) acetamide, selectively protects against oxidative stress-induced cell death by activating the Nrf2-ARE pathway: therapeutic implications for ALS. Free Radic. Biol. Med. 53(11), 2028-2042 (2012).

12 Jimenez-Sanchez M, Licitra F, Underwood BR et al. Huntington's disease: mechanisms of pathogenesis and therapeutic strategies. Cold Spring Harb. Perspect. Med. doi:10.1101/ cshperspect.a024240 (2016) (Epub ahead of print).

13 Johri A, Beal MF. Mitochondrial dysfunction in neurodegenerative diseases. J. Pharmacol. Exp. Ther. 342(3), 619-630 (2012).

14 Jazwa A, Rojo AI, Innamorato NG et al. Pharmacological targeting of the transcription factor Nrf2 at the basal ganglia provides disease modifying therapy for experimental parkinsonism. Antioxid. Redox Signal. 14(12), 2347-2360 (2011).

15 Ahuja M, Kaidery AN, Yang L et al. Distinct Nrf2 signaling mechanisms of fumaric acid esters and their role in neuroprotection against 1-methyl-4-phenyl-1,2,3,6 tetrahydropyridine-induced experimental Parkinson's-like disease. J. Neurosci. 36(23), 6332-6351 (2016).
16 Lastres-Becker I, García-Yagüe AJ, Scannevin $\mathrm{RH}$ et al. Repurposing the NRF2 activator dimethyl fumarate as therapy against synucleinopathy in Parkinson's disease. Antioxid. Redox Signal. 25(2), 61-77 (2016).

17 Liu Y, Deng Y, Liu H et al. Hydrogen sulfide ameliorates learning memory impairment in APP/PS1 transgenic mice: a novel mechanism mediated by the activation of $\mathrm{Nrf2}$. Pharmacol. Biochem. Behav. 150-151, 207-216 (2016).

18 Fox RJ, Kita M, Cohan SL et al. BG-12 (dimethyl fumarate): a review of mechanism of action, efficacy, and safety. Curr. Med. Res. Opin. 30 (2), 251-262 (2014).

19 Bertrand HC, Schaap M, Baird L et al. Design, synthesis, and evaluation of triazole derivatives that induce Nrf2 dependent gene products and inhibit the Keap1-Nrf2 protein-protein interaction. J. Med. Chem. 58(18), 7186-7194 (2015).

20 Quinti L, Casale M, Moniot S et al. SIRT2- and NRF2-targeting thiazolecontaining compound with therapeutic activity in Huntington's disease models. Cell. Chem. Biol. 23(7), 849-861 (2016). 